\title{
Spacetime Processing Metasurfaces: GSTC Synthesis and Prospective Applications
}

\author{
Nima Chamanara, Yousef Vahabzadeh, Karim Achouri, and Christophe Caloz \\ Polytechnique Montréal, \\ Montréal, Québec H3T 1J4, Canada. \\ e-mail: nima.chamanara@polymtl.ca
}

\begin{abstract}
The paper presents the general concept of spacetime processing metasurfaces, synthesized by generalized sheet transition conditions (GSTCs). It is shown that such metasurfaces can perform multiple simultaneous spatio-temporal processing transformations on incident electromagnetic waves. A time-reversal space-generalized-refraction metasurface and multi-time-spacedifferentiating metasurfaces are presented as applications of the general spacetime processing metasurface concept.

Index Terms-Metasurface, generalized sheet transition conditions (GSTCs), spacetime transformations, time-varying medium, time reversal, generalized refraction, time differentiator.
\end{abstract}

\section{INTRODUCTION}

Metasurfaces are periodic or aperiodic arrays of scattering elements arranged in a subwavelengthly thin substrate [1]. They transform electromagnetic waves in unusual ways and they have already been demonstrated in a diversity of applications, including polarization transformers and rotators [2], [3], perfect absorbers [4], beam transformers [5], electromagnetic interferometers and transistors [6] and nonreciprocal metasurfaces [3], [7]-[9].

Designing metasurfaces is generally a challenging inverse problem. Synthesis techniques include heuristic approaches, and more general techniques such as momentum transformation [5] and the general sheet transition condition (GSTC) synthesis approach [10], [11]. The latter, that provides the general bianisotropic surface susceptibility tensors of the metasurface, is a powerful design technique: 1) It is exact; 2) It is general, transforming arbitrary incident waves into arbitrary reflected and transmitted waves, 3) it often admits closedform solutions, 4) it provides deep insight into the physics of the transformations, 5) it allows multiple (at least up to 4) simultaneous and independent transformations. However, this method has been so far restricted to monochromatic and space-only-varying wave transformations [11].

This paper extends the GSTC susceptibility technique to space-and-time-varying metasurfaces and presents two corresponding applications. Moreover, it is noted that such a spacetime-varying metasurface also applies to the spatiotemporal processing of multiple simultaneous waves.

The organization of the paper is as follows. The GSTC approach of spacetime-varying metasurfaces is described in Sec. III Two illustrative examples are presented in Sec. III] Conclusions are given in Sec. IV.

\section{Time-VARying GSTC-BASEd Metasurfaces}

Metasurfaces can be effectively modeled as a zero-thickness sheet that creates a discontinuity in the electromagnetic field. Such a discontinuity can be most generally described by generalized sheet transitions conditions (GSTCs) [10]. In the time domain, GSTCs read

$$
\begin{gathered}
\mathbf{n} \times\left(\mathbf{H}^{+}-\mathbf{H}^{-}\right)=\mathbf{J}_{\mathbf{0}}+\frac{\partial}{\partial t} \mathbf{P}_{0 \mathrm{t}}-\frac{1}{\mu_{0}} \mathbf{n} \times \nabla M_{0 \mathrm{n}}, \\
\mathbf{n} \times\left(\mathbf{E}^{+}-\mathbf{E}^{-}\right)=-\mathbf{K}_{\mathbf{0}}-\frac{\partial}{\partial t} \mathbf{M}_{0 \mathrm{t}}-\frac{1}{\epsilon_{0}} \mathbf{n} \times \nabla P_{0 \mathrm{n}},
\end{gathered}
$$

where the + and - superscript denote the fields specified on both sides of the metasurface (-: incident and reflected, $+:$ transmitted), where the $\mathrm{t}$ and $\mathrm{n}$ subscripts denote the tangential and normal components, respectively, and where the 0 subscript denote the first-order Dirac delta terms of the 2D discontinuity.

The conditions (1) and (2) are valid for any time-continuous electromagnetic waves. Therefore, one may specify arbitrary time-varying, in addition to spatially-discontinuous, electromagnetic fields on the two sides of the metasurface, $\mathbf{E}^{ \pm}(\mathbf{r}, t)$ and $\mathbf{H}^{ \pm}(\mathbf{r}, t)$. As a result, one finds the corresponding timevarying, in addition to space-varying, electric and magnetic polarization densities that satisfy these conditions $\mathbf{P}_{0 \mathrm{t}}(\mathbf{r}, t)$ and $\mathbf{M}_{0 \mathrm{t}}(\mathbf{r}, t)$. The time-varying and space-varying susceptibilities, $\overline{\bar{\chi}}_{\mathrm{uv}}(\mathbf{r}, t)$ where uv represents the 4 combinations ee, $\mathrm{mm}$, em and me, can then be found through the averages of the electric and magnetic fields on the metasurface at each time instant, following a similar procedure as in [11].

\section{ILlustrative EXAMPLES}

A spacetime-varying metasurface can in principle arbitrarily transform the spatial and temporal frequency contents of any incident electromagnetic wave. We shall present here two illustrative application examples.

The first application is depicted in Fig. 1 A chirped Gaussian electromagnetic pulse is impinging on the spacetimevarying metasurface and the metasurface is specified to transform this wave into a reversed-chirped wave refracted in a different direction, and with zero reflection. The corresponding 
spacetime-varying metasurface susceptibility tensors are found following the procedure outlined in the previous section, leading exactly to the transformation shown in the figure. Note that the spatio-temporal frequency contents of the input chirp are simultaneously transformed by the metasurface: the normally incident electromagnetic pulse is time reversed (temporal frequency transformation) and transmitted at an oblique angle (spatial frequency transformation).

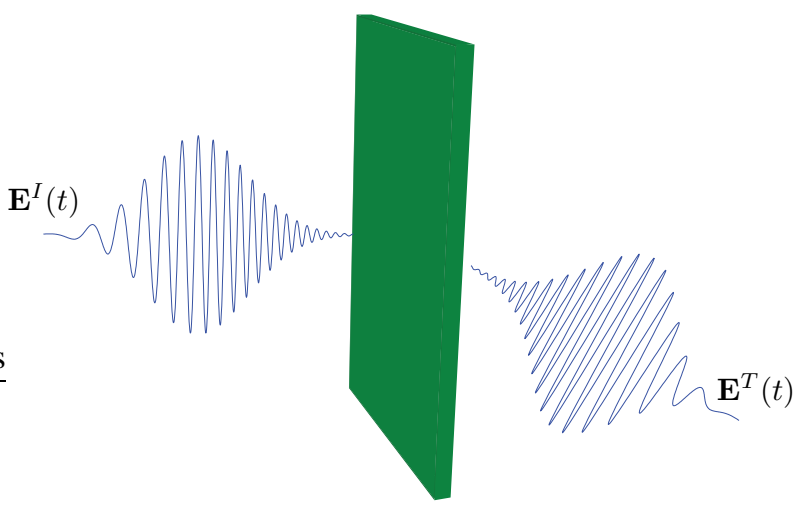

Fig. 1. Time-reversal generalized-refractive spacetime metasurface. A normally incident chirped pulse is time-reversed and refracted under an oblique angle.

The second application is depicted in Fig. 2, It represents a metasurface that is a temporal differentiator and a spatial splitter. Specifically, the metasurface transforms a normally incident electromagnetic wave in the following fashion: it generates the first time-derivative of part of the wave and radiates the resulting wave in a specified direction, and generates the second time-derivative of the rest of the wave, that it radiates in another specified direction. In this way, detectors placed in the corresponding directions receive the two derivatives, in realtime. Such a multiple-transformation system is possible, again following the procedure described in the previous section, if the susceptibility tensors include a sufficient number of degrees of freedom [11].

\section{CONClusions}

Spacetime processing metasurfaces obtained by generalized sheet transition conditions (GSTCs) susceptibility synthesis have been presented. It has been shown that such metasurfaces could perform multiple simultaneous spatio-temporal processing transformations on incident electromagnetic waves. Two examples have been shown: a time-reversal space-refractor metasurface and time-space differentiator metasurface. Such spacetime metasurfaces require active elements and may thereby also provide gain and reconfigurability [12].

\section{REFERENCES}

[1] C. Holloway, E. F. Kuester, J. Gordon, J. O'Hara, J. Booth, and D. Smith, "An overview of the theory and applications of metasurfaces: the twodimensional equivalents of metamaterials," IEEE Antennas Propag. Mag., vol. 54, pp. 10-35, April 2012.

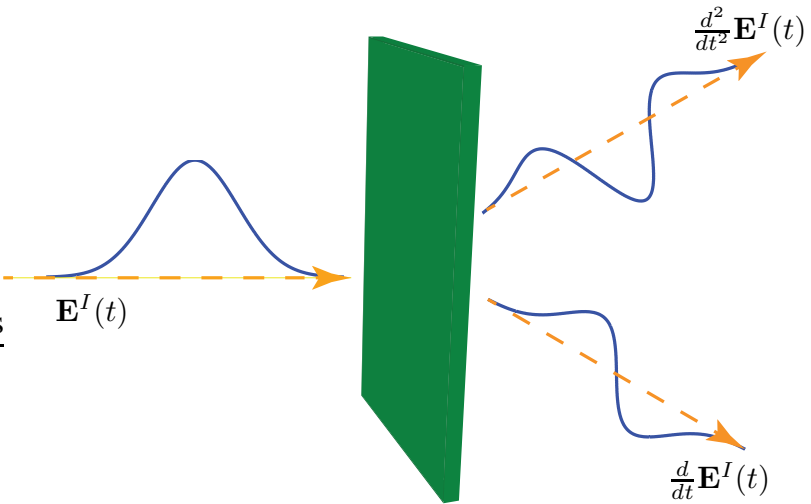

Fig. 2. Differentiator spacetime metasurface. A normally incident Gaussian pulse is time-differentiated and different time derivatives are transmitted to different specified directions.

[2] T. Niemi, A. O. Karilainen, S. Tretyakov, et al., "Synthesis of polarization transformers," Antennas Propag. IEEE Trans. on., vol. 61, no. 6, pp. 3102-3111, 2013.

[3] T. Kodera, D. L. Sounas, and C. Caloz, "Artificial faraday rotation using a ring metamaterial structure without static magnetic field," Appl. Phys. Lett., vol. 99, no. 3, p. 031114, 2011.

[4] Y. Ra'di, V. S. Asadchy, S. Tretyakov, et al., "Total absorption of electromagnetic waves in ultimately thin layers," Antennas Propag. IEEE Trans. on., vol. 61, no. 9, pp. 4606-4614, 2013.

[5] M. A. Salem and C. Caloz, "Manipulating light at distance by a metasurface using momentum transformation," Opt. express., vol. 22, no. 12, pp. 14530-14543, 2014.

[6] K. Achouri, G. Lavigne, M. A. Salem, and C. Caloz, "Metasurface spatial processor for electromagnetic remote control," arXiv preprint arXiv:1510., 2015.

[7] D. L. Sounas, T. Kodera, and C. Caloz, "Electromagnetic modeling of a magnet-less non-reciprocal gyrotropic metasurface," Trans. Antennas Propag., vol. 61, pp. 221-231, Jan. 2013.

[8] Y. Hadad, D. Sounas, and A. Alu, "Space-time gradient metasurfaces," Phys. Rev. B., vol. 92, no. 10, p. 100304, 2015.

[9] B. A. Khan, S. Gupta, and C. Caloz, "Spatial nonreciprocal and nongyrotropic structure," (Hobart, Australia), pp. 154-157, Nov. 2015.

[10] M. Idemen and A. H. Serbest, "Boundary conditions of the electromagnetic field," Electron. Lett., vol. 23, no. 13, pp. 704-705, 1987.

[11] K. Achouri, M. Salem, and C. Caloz, "General metasurface synthesis based on susceptibility tensors," IEEE Trans. Antennas Propag., 2014.

[12] S. Gupta and C. Caloz, "Perfect dispersive medium," arXiv:1511., Nov. 2015. 